\title{
A Flexible Path for Human and Robotic Space Exploration
}

\author{
David J. Korsmeyer ${ }^{1}$ and Robert R. Landis ${ }^{2}$ \\ NASA Ames Research Center, Moffett Field, CA, 94035, USA \\ R. Gabriel Merrill ${ }^{3}$ and Daniel D. Mazanek ${ }^{4}$ \\ NASA Langley Research Center, Hampton, VA, 23681, USA \\ Robert D. Falck ${ }^{5}$ \\ NASA Glenn Research Center, Cleveland, OH, 44111, USA \\ Robert B. Adams ${ }^{6}$ \\ NASA Marshall Space Flight Center, Huntsville, AL, 35812, USA
}

\begin{abstract}
During the summer of 2009, a flexible path scenario for human and robotic space exploration was developed that enables frequent, measured, and publicly notable human exploration of space beyond low-Earth orbit (LEO). The formulation of this scenario was in support of the Exploration Beyond LEO subcommittee of the Review of U.S. Human Space Flight Plans Committee that was commissioned by President Obama. Exploration mission sequences that allow humans to visit a wide number of inner solar system destinations were investigated. The scope of destinations included the Earth-Moon and Earth-Sun Lagrange points, near-Earth objects (NEOs), the Moon, and Mars and its moons. The missions examined assumed the use of Constellation Program elements along with existing launch vehicles and proposed augmentations. Additionally, robotic missions were envisioned as complements to human exploration through precursor missions, as crew emplaced scientific investigations, and as sample gathering assistants to the human crews. The focus of the flexible path approach was to gain ever-increasing operational experience through human exploration missions ranging from a few weeks to several years in duration, beginning in deep space beyond LEO and evolving to landings on the Moon and eventually Mars.
\end{abstract}

\section{NASA Support of the Review of U.S. Human Space Flight Plans Committee}

$\mathrm{O}$ May 7, 2009, the White House Office of Science and Technology Policy (OSTP) asked the National Aeronautics and Space Administration (NASA) to initiate an independent review of ongoing U.S. human space flight plans and programs, as well as alternatives, to ensure that the U.S. was pursuing the best strategy for the future of human space flight - one that was safe, innovative, affordable, and sustainable. Led by aerospace businessman Norman Augustine, the Review of U.S. Human Space Flight (HSF) Plans Committee was asked to identify and characterize a reasonable range of architectural options for continuation of U.S. human space flight activities beyond retirement of the Space Shuttle. The identification and characterization of these options were to address the following objectives: a) expediting a new U.S. capability to support utilization of the International Space Station (ISS); b) supporting missions to the Moon and other destinations beyond low-Earth orbit (LEO); c) stimulating commercial space flight capability; and d) fitting within the current NASA budget profile for human space exploration activities.

In addition to the objectives described above, the review examined and assessed the appropriate amount of research and development and complementary robotic activities needed to make human space flight activities most productive and affordable over the long term, as well as appropriate opportunities for international collaboration. Finally, the review evaluated options for extending ISS operations beyond 2016, and most importantly it evaluated what capabilities would be enabled by each of the potential architectures considered (Ref. 1).

\footnotetext{
${ }^{1}$ Chief, Intelligent Systems Division, Mail Stop 269-1, AIAA Associate Fellow.

${ }^{2}$ New Mission Concepts lead, Intelligent Systems Division, Mail Stop 269-1, AIAA Member.

${ }^{3}$ Aerospace Systems Engineer, Space Missions Analysis Branch, Mail Stop 462, AIAA Member .

${ }^{4}$ Aerospace Systems Engineer, Systems Analysis \& Concepts Directorate, Mail Stop 462, AIAA Senior Member.

${ }^{5}$ Aerospace Engineer, Mission Design \& Analysis Branch, Mail Stop 105-3.

${ }^{6}$ Advanced Propulsion Technologist, Advanced Concepts Office, Bldg 4487/C226, AIAA Senior Member
} 


\section{A. NASA's Engineering Analysis Team Support}

The Review of U.S. Human Space Flight Plans Committee (subsequently referred to as "HSF Committee" in this paper) requested that NASA provide support for the committee's activities. NASA headquarters provided policy documents and access to all available information for historical and current programs. In particular, the committee desired specific engineering and analysis to support their review. In order to respond to this engineering request, the NASA Engineering and Safety Center's (NESC) took the lead role for organizing NASA's support. Representatives from all of the NASA Centers were selected and the Engineering Analysis (EA) Team was formed. NASA established an online document and data repository and the EA Team provided many reports and initial analyses for the committee members as they reviewed NASA's current work and the missions that NASA had assessed over the past several decades. Finally, several subcommittees were created to address various areas of interest for human exploration and to perform specific assessments. Figure 1 describes the scope of each subcommittee and identifies each of its members.

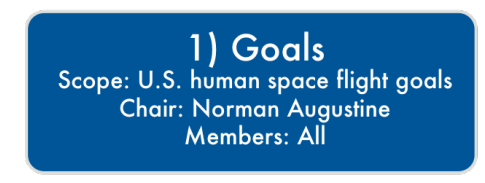

subcommittees
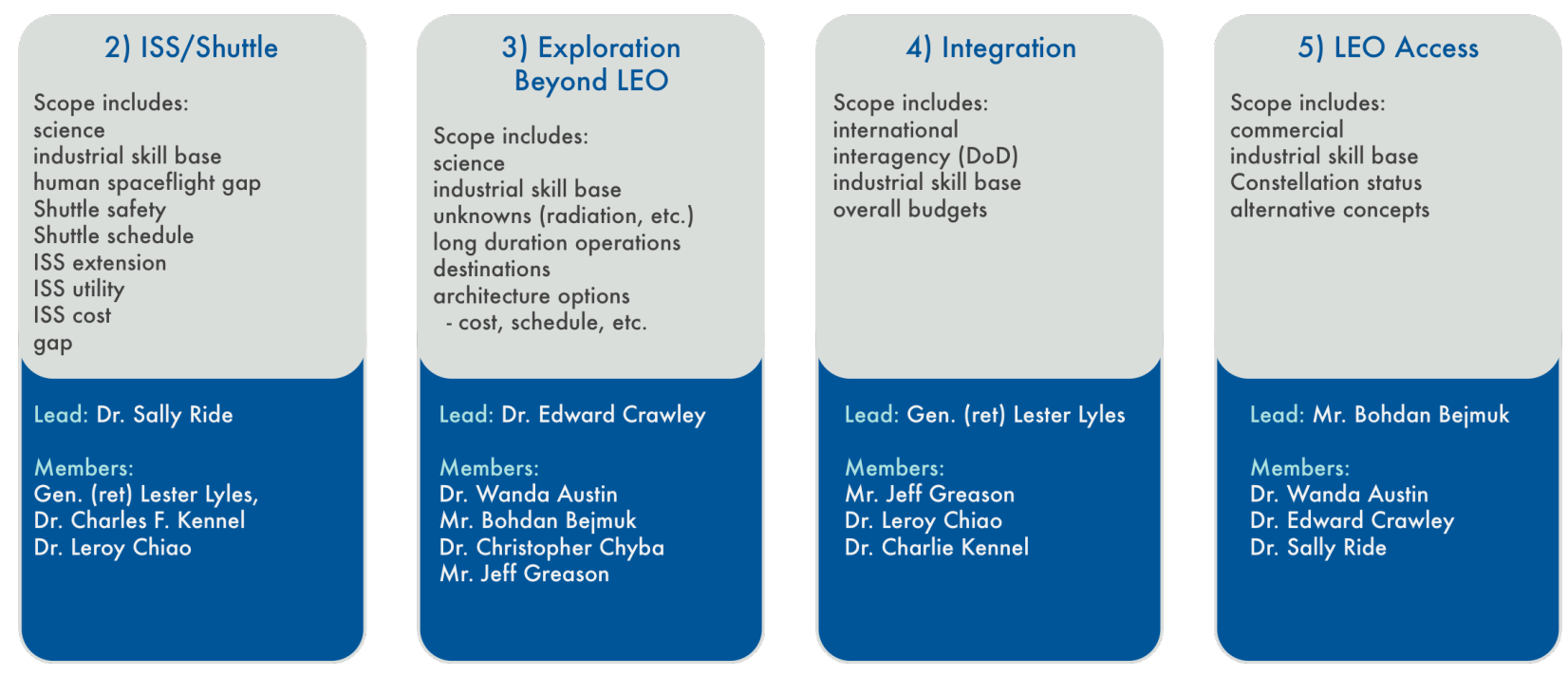

Figure 1 - HSF Subcommittee's Scope and Membership

\section{B. Exploration Beyond LEO Subcommittees and the Five Scenarios Investigated}

Dr. Edward Crowley, Professor of Aeronautics and Astronautics, and the Engineering Systems Ford Professor of Engineering, at the Massachusetts Institute of Technology (MIT), was the subcommittee lead for architectures that focused on exploration beyond low-Earth orbit. The Exploration Beyond LEO subcommittee proposed the following five exploration scenarios for detailed analysis by the NASA EA Team:

- Scenario A: Lunar Base

This scenario is a minor simplification of the NASA Constellation Program lunar outpost capability, and is intended to form a baseline that is close to the way the reference program would probably end up in a few years under continued budget pressure. It is intended to focus on a polar lunar base, and does not have the requirements for anytime Earth return and global lunar access.

- Scenario B: Lunar Global

This scenario is intended as a lunar exploration approach that investigates significant alternatives to the NASA Constellation Program lunar outpost capability. It utilizes self-contained, and potentially mobile, outposts to provide surface habitation (as was studied by the NASA Lunar Architecture Team-2 in 2007). The scenario elements are not specifically applicable to Mars exploration, but the scenario lays the technology groundwork for Mars missions by developing subsystems and technologies that are extensible to Mars. 


\section{- $\quad$ Scenario C: Moon to Mars}

This scenario captures a third possible rationale for going to the Moon - literally to use it as a minimalist part of developing the technical and programmatic capability to explore Mars with humans. The Moon missions function primarily as a test program for verifying key elements of the capability needed to send humans to Mars. Lunar science and exploration are simply targets of opportunity, while the real scientific and exploration target is assumed to be Mars.

\section{- $\quad$ Scenario D: Mars First}

This scenario advocates bypassing exploration of the Moon completely, and sending a crewed mission to Mars by developing a "lower cost" approach than those used in the NASA Mars Design Reference Architecture (DRA). For example, this approach would rely more heavily on In-Situ Resource Utilization (ISRU) for both Environmental Control and Life Support System (ECLSS) and propulsion fuel and oxidizers (both Mars assent and trans-Earth injection). This scenario is intended to develop and compare the benefits and costs of this approach on an even basis with the other scenarios.

\section{- Scenario E: Flexible Path}

The intent of this scenario it to present an alternative exploration approach by developing the capabilities and assets for human exploration that will make substantive exploration progress that engages the public and returns significant scientific knowledge, while delaying the development of the descent systems (i.e., a lunar lander) and surface infrastructure required in the other scenarios. This scenario would also focus on the effects of radiation exposure and protection methods as a primary enabler of human space exploration, by having missions of progressively longer duration outside of the Earth's magnetosphere.

In all of the scenarios outlined by the Exploration Beyond LEO subcommittee, the following common assumptions and constraints were applied and are addressed:

- Assume a reference crew of four for the Moon and Mars, and estimate sensitivity to as few as two for the Moon, and as many as six crew-members for Mars.

- Assume the use of baseline Constellation Program elements along with existing and augmented launch vehicles along with proposed space-based infrastructure elements (i.e., Orion Crew Exploration Vehicle, Ares I and Ares V launch vehicles, 25, 75 and 125 metric ton to LEO launchers, propellant depots in LEO and/or at Earth-Moon Lagrange points).

- Track the total initial mass in LEO (IMLEO) along with the smallest practical mass and shroud diameter for LEO delivery systems. Report whether a particular scenario is feasible under the various transportation infrastructure assumptions considered.

- Assume technology investments that can be made to increase Technology Readiness Level (TRL) from TRL 2 and above to the appropriate TRL for a particular scenario implementation, and identify and report these necessary investments (Ref. 2).

- Track the assumptions about robotic exploration, and associated investments, that are built into the scenario.

\section{A Flexible Path of Exploration}

The Flexible Path exploration approach allows humans to visit a wide range of inner solar system destinations, but not go to the surface of those with deep gravity wells, including the Moon, Mars and possibly Venus, as shown in Figure 2. Principal destinations would include the Earth Moon L1 and L2 Lagrange points, the Earth Sun L1 and L2 Lagrange points, near-Earth objects (NEOs) and the moons of Mars. The Flexible Path scenario's goal is to allow progressively more challenging human exploration missions and to provide exciting new scientific discoveries each step of the way. The emphasis would be obtaining multi-kilogram samples from a variety of solar system planetary bodies through telerobotic exploration and direct human interaction with the target. In the case of the larger planetary bodies of the Moon and Mars, the humans would remain in orbit, deploy probes, and teleoperate surface robotic vehicles, including rendezvousing with sample returns missions sent from the surface. In the case of smaller objects such as NEOs, the crew would explore the surfaces directly and return samples. This scenario in no way precludes future surface exploration at some later time, simply that this approach would first exploit all exploration that could be accomplished without landing on a planetary surface. Additional Flexible Path missions can include human lunar surface exploration and Mars landings as NASA funding profiles permit.

A key benefit of this architecture is development of a NASA and contractor workforce that will have engineered and operated increasing complex and robust missions and systems for human exploration. During the 1960's "Moon race", NASA conducted increasingly more challenging missions starting with Project Mercury and Project Gemini, and culminating with the Apollo Program that successfully landed six crews on the lunar surface and returned them to Earth. In a similar fashion, the missions envisioned in the Flexible Path architecture sequence build incrementally upon each prior mission's capabilities and permit a sustainable level of human space exploration. 


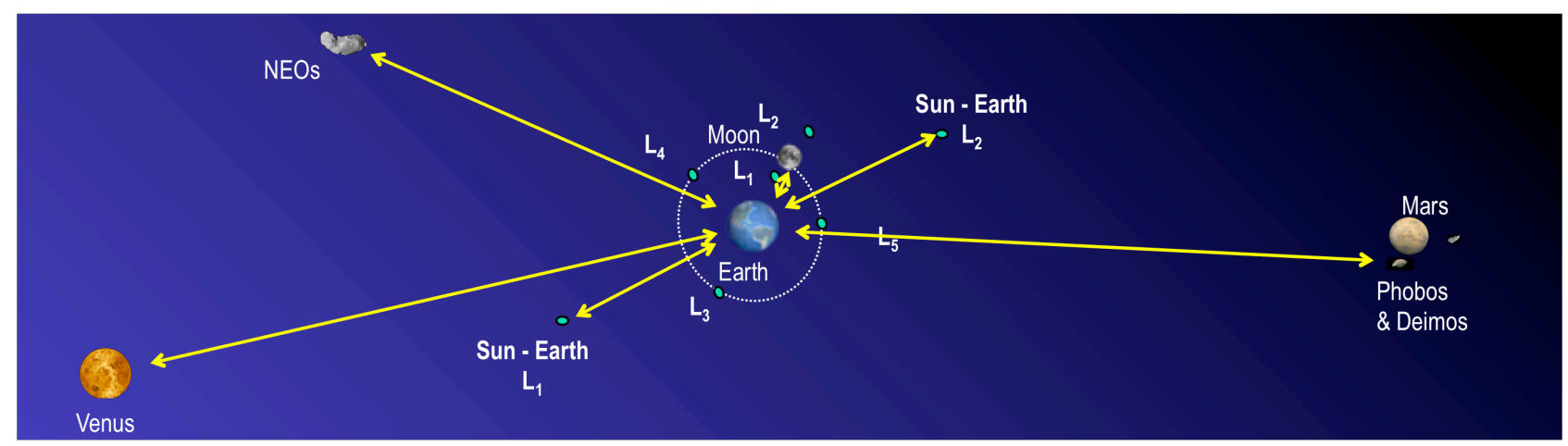

Figure 2 - Destination Scope for the Flexible Path Architecture

In addition, the critical scientific components of human spaceflight will be addressed through incrementally more aggressive exploration missions into the inner-solar system. Determining the human physiological and operational impacts and countermeasures due to long-term radiation environment, including galactic cosmic rays (GCRs) and Solar Particle Events (SPEs), and extended exposure to zero-gravity is necessary to a sustained capability for human exploration.

A sustained exploration program by the United States requires continual public engagement, inspiration, and benefit, while operating within the fiscal constraints of the NASA budget and including contributions from our international partners. The Flexible Path missions are designed to cultivate and maintain public support and interest in human spaceflight by taking on demonstrably new, visible, and different missions. This encompasses unprecedented deep space missions with dramatic perspectives of humankind's home planet as a member of the inner solar system including NEOs, the Moon, and Mars.

\section{A. Scenario Ground Rules adopted by the NASA Flexible Path Study Team}

There are many possible approaches to implementing a Flexible Path architecture. The Flexible Path Study Team was required to develop the scenario under the assumptions and constraints provided by the Exploration Beyond LEO Subcommittee. A key assumption for the Flexible Path scenario described in this paper was the use of baseline Constellation Program elements along with existing launch vehicles and proposed augmentations. Additionally, it was assumed that viable and relevant exploration missions can be completed with a single crew launch along with chemical and nuclear thermal in-space propulsion stages. As additional deep space capabilities are developed and become operational, the scope of potential missions increases. Certain enabling technologies are assumed available in order for Flexible Path missions to be performed. First among those is a cryogenic (liquid oxygen/liquid hydrogen) in-space propulsion stage able to have a near zero (just a few percent) boil-off of propellant over approximately 200 days. The second most important technology development is a high-performance in-space engine capable of multiple restarts. Additional enabling technologies include in-space cryogenic fluid transfer, radiation shielding and countermeasures, improved regenerative life support systems, technologies for deep-space operational autonomy (in both crewed and uncrewed modes), and telerobotic systems to be operated by the crew in deep space. It was assumed that a habitation module (either rigid or inflatable) can be procured from a commercial entity, alleviating NASA from the majority of the development costs. Robotic missions are complementary and require some technical development. In particular, a Mars sample return mission could utilize the in-space propulsion stage designed for human missions to send multiple sampling missions to Mars, and the Mars-entry aeroshell utilized could validate technologies for eventual human landings.

The Flexible Path Study team assumed a baseline Orion block II Crew Exploration Vehicle (CEV) capable of accommodating up to four crewmembers for durations up to 18 days. All of the Earth entry, descent, and landing was assumed to fall within the nominal design requirements for the Orion Crew Module (CM). All trajectories analyzed assumed a maximum Earth atmosphere entry velocity of $11 \mathrm{~km} / \mathrm{s}$. Since the Orion CM cannot support the crew's extravehicular activities (EVAs) without full cabin depressurization, the team decided to add a small combined airlock/habitation module to provide additional pressurized and habitable volume for Earth-Moon and Sun-Earth Lagrange point and initial NEO missions. Three classes (50 t, $75 \mathrm{t}$, and $125 \mathrm{t}$ ) of in-space propulsion stages were assumed available for each mission in the Flexible Path sequence. The mission concept of operations determined the required cargo mass, and that subsequently drove the required propellant loading and the number of stages required for each mission. Missions were constrained by the team to only use up to two stages of a given tonnage class to minimize staging operational complexity. 


\section{B. Initial Flexible Path Sequence and Destinations}

In order to give NASA some guidance beyond the simple destinations described so far in this paper, the Exploration Beyond LEO subcommittee provided a sequence of notional missions for the Flexible Path scenario. While the different Flexible Path missions can be assembled in almost any order, a logical sequencing allows missions to build upon the experience gained from prior missions. Additionally, several missions require less hardware and systems to be developed. These considerations, accompanied by the desire to have each mission reduce technical and operation risk for the next mission, resulted in the following nominal sequence being proposed. The NASA Flexible Path Study Team took the initial mission sequence, along with constraints and ground rules provided by the subcommittee, and identified the missions that were analyzed. While additional Flexible Path missions can be envisioned (e.g., geostationary satellite servicing), these initial missions and their sequencing provided the basis to perform a preliminary feasibility study. Note that all of these missions shown below and in Figure 3 are assumed to be crewed, except as noted.

\section{A Nominal Sequence for Flexible Path Exploration:}

1. An uncrewed circumlunar mission utilizing only the Orion CEV and an in-space propulsion stage.

2. A circumlunar mission only the Orion CEV and an in-space propulsion stage.

3. A mission to the Earth-Moon L1 Lagrange point, to demonstrate the ability to perform crewed operations and emplace and service assets stationed there.

4. A mission to the Sun-Earth L2 Lagrange point, to demonstrate the ability to emplace and service scientific assets stationed there.

5. A mission to the Sun-Earth L1 Lagrange point for additional deep-space exploration preparation (i.e., experience with the interplanetary radiation environment outside the Earth's magnetosphere, which is not the case at the Sun-Earth L2 point) and to emplace and service of science scientific assets stationed there.

6. Several missions to rendezvous with NEOs of different composition (e.g., metallic, carbonaceous chondrites, etc.) for exploration, scientific instrument emplacement, and the return of samples.

7. A free return mission to Mars, with a fly-by, but no major maneuvering in the vicinity of Mars.

8. A mission to the moons of Mars (Phobos and/or Deimos) combined with the return of samples from a robotic mission to the Martian surface.

These Flexible Path missions culminate with the capability to deliver humans to an orbit around Mars and return them safely to Earth. At this point the next logical step is to develop the systems and technologies to land humans on the Martian surface prior to exploring outside the inner solar system. An additional "off-ramp" for the Flexible Path mission sequence could focus on exploring the lunar surface to gain the needed technical and operational experience associated with human and robotic surface exploration.

\section{Flexible Path Scenario $E$ is a sequence of missions with increasing capability into the inner solar system}

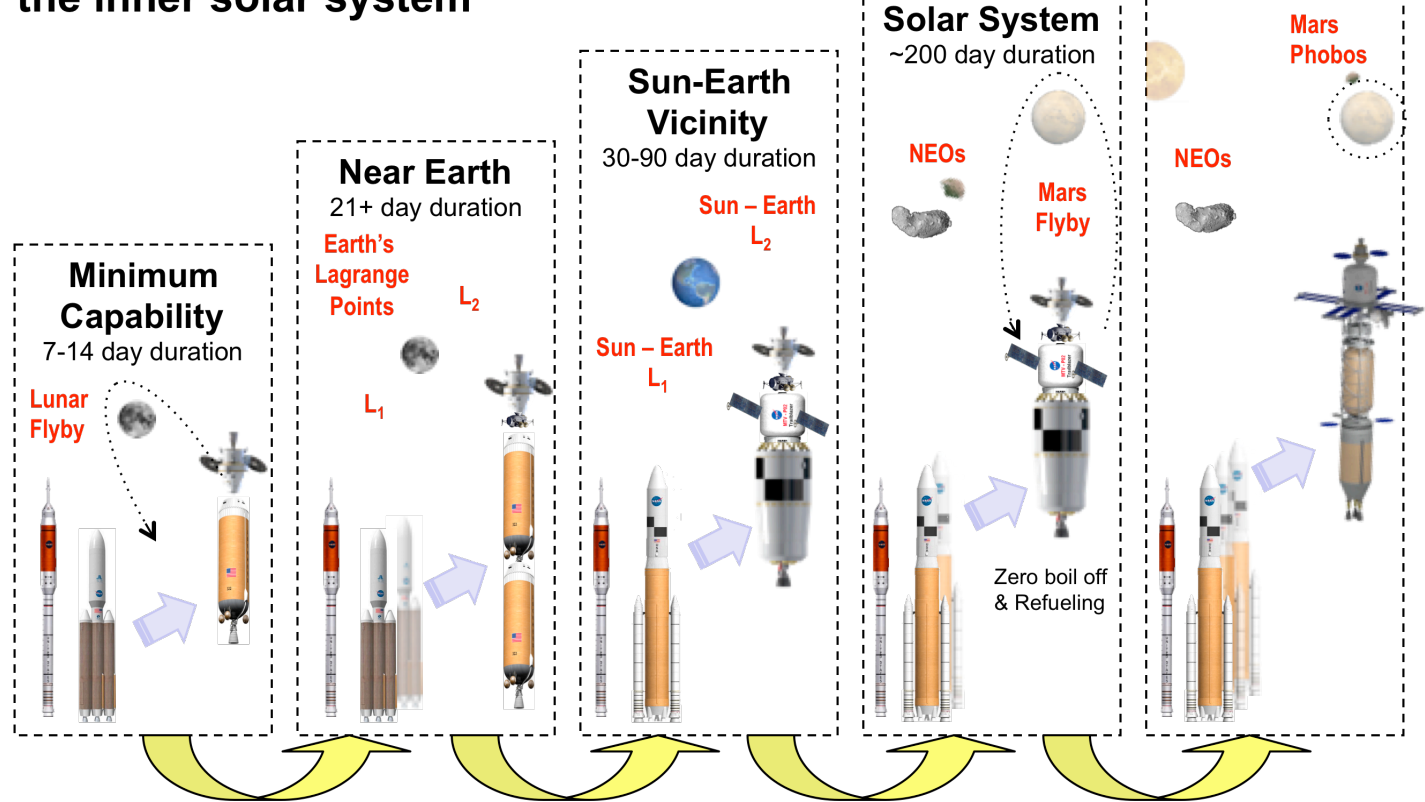

Figure 3 - Nominal Milestones and Sequence for Flexible Path Missions

\section{Inner}

Solar System

Solar System

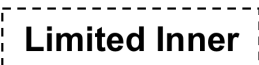

Limited Inner

Solar System

(n)




\section{Flexible Exploration Destination Mission Descriptions}

The Flexible Path scenario is composed of mission destinations that provide incrementally increasing engineering and operational difficulty for ground personnel and the mission crews, provide unique scientific return, and engage and excite the public. The following descriptions provide an overview of each of the Flexible Path mission concepts.

\section{A. Lunar Free-Return Missions}

The goal of the lunar free-return mission in the Flexible Path architecture is to provide the first operational test of the Orion CEV systems outside of LEO. Since no major propulsive maneuvers are required after trans-lunar injection (TLI), missions launched into a free-return trajectory around the Moon allow the safe return of the spacecraft in the event of a failure of the main propulsion system after TLI (Ref. 3). This mission is analogous to the Apollo 8 lunar mission that tested out the Command and Service Modules while enabling the first humans to orbit the Moon. Apollo 8 was the first human spaceflight mission to escape from the gravitational field of the Earth, and the first crewed mission to return to Earth from another celestial body.

\section{B. Earth-Moon Lagrange Point Missions}

Missions to the Earth-Moon (E-M) and Earth-Sun (E-S) Lagrange points offer a good stepping-stone to deep-space missions of longer duration. These missions offer a way of testing vehicle subsystems in an environment outside of the protection of the Earth's magnetic field in conjunction with relatively low mission velocity change (DV or $\Delta \mathrm{V})$ and relatively short trip times. The orbital mechanics are not tightly coupled to time, so an early return is feasible should problems arise.

There are several potential applications of the architecture at the Lagrange points. Missions to the Earth-Moon Lagrange point have been proposed in several NASA studies with several proposing propellant re-supply or even E-M L1 gateways for Mars exploration (Ref. 4).
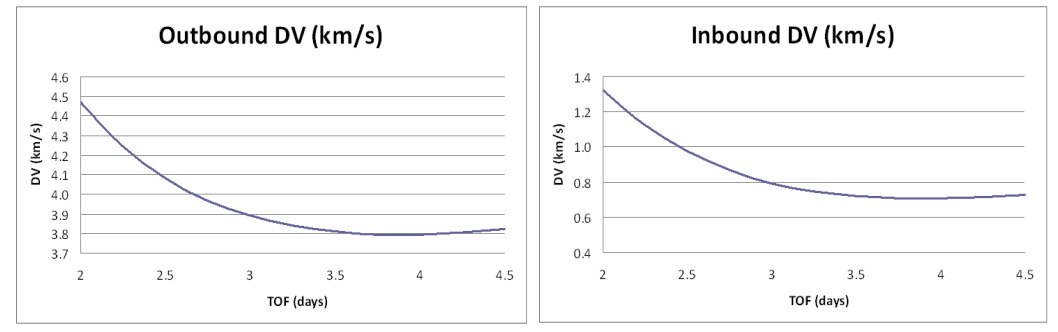

Figure 4 - Earth- Moon L1 Trajectory Curves

There are unique science opportunities at E-M L1 due to the nearly continuous full-sky viewing and true deep-space environment while still inside the terrestrial magnetosphere for radiation protection. The E-M L1 location provides a continuous view of lunar nearside, of the Earth, and allows the testing of free-space orbital operations with no atmospheric drag and no relative orbital rotations as in LEO operations. This mission can provide a deep-space human exploration analog with relatively easy return to earth. Additionally, the E-M L1 point provides global anytime lunar access for little additional energy. It also enables very low energy transfers available between libration points. It would be possible to assemble and deploy robotic science platforms (e.g., advanced astronomical telescopes) at the E-M L1 location and then transfer the platforms, with only a $14 \mathrm{~m} / \mathrm{s}$ velocity change, from the Earth-Moon L1 point to a EarthSun L2 halo orbit for operation.

Trajectory analysis of Earth-Moon L1 was run and results were combined to generate curves that provide the $\Delta \mathrm{V}$ required for either an outbound or inbound trip, as shown in Figure 4. The time of flight from the Earth to Earth-Moon Lagrange points is short, with travel times between 3 and 4 days being the most efficient. Injection and insertion energy required for these trajectories is similar to low those required for lunar orbital missions.

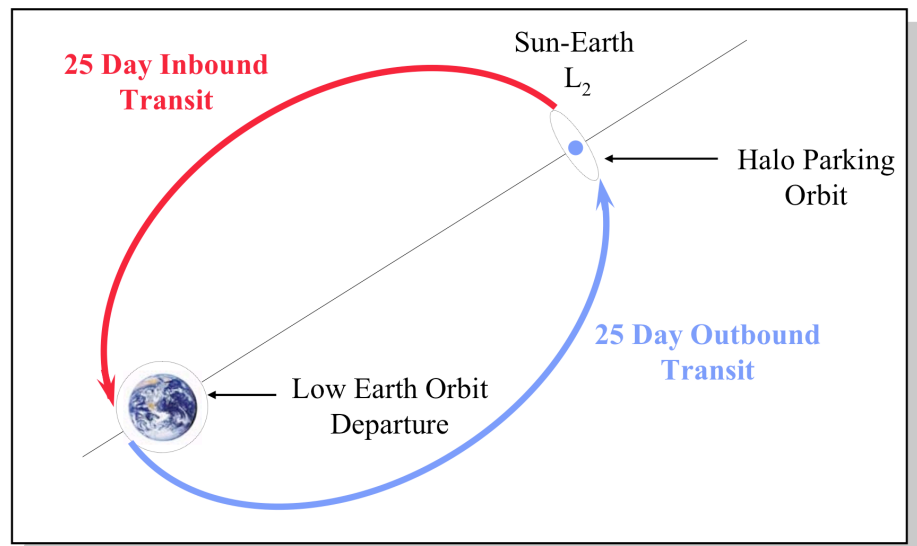

Figure 5 - Sun-Earth L2 Concept Trajectory

\section{Sun-Earth Lagrange Point Missions}

Human missions to the Sun-Earth Libration Points can provide a fairly inexpensive and early validation of core exploration capabilities and technologies (Ref. 5). These include a deep-space airlock for the Orion CEV, a high-performance cryogenic chemical propulsion stage, and possibly a separate habitation module. Returning from the Sun-Earth Lagrange points will also allow for a test of the reentry systems from interplanetary space. These missions will be the first demonstration of interplanetary spacecraft hardware and systems, and will increase the deepspace operational experience of the ground 
personnel and mission crews. It is critical for NASA to bridge the gap from the operational experience of conducting LEO missions and the changes required for long-duration, deep-space missions where communications are significantly time-delayed. Other potential applications of such missions are servicing or emplacing of robotic science assets at the Sun-Earth L1 and L2 points and the utilization of quasi-stable halo orbits about such points for future propellant depots and other infrastructure.

The Sun-Earth L2 location is an excellent location for space-based observatories as they maintain the same orientation with respect to both the Sun and the Earth. A Sun-Earth L2 concept trajectory is shown in Figure 5. The Wilkinson Microwave Anisotropy Probe, Herschel Space Observatory and Planck space observatory are in orbit around the Sun-Earth L2 point. The Gaia probe and James Webb Space Telescope will also be placed at the Sun-Earth L2.

The Sun-Earth L1 location is ideal for observations of the Sun, with the Solar and Heliospheric Observatory (SOHO) and the Advanced Composition Explorer (ACE) both at the Sun-Earth L1 point. A mission to Sun-Earth L1 will provide crew and systems experience with the interplanetary radiation environment outside the Earth's magnetosphere, which is not the case at Sun-Earth L2 point. As with a Sun-Earth L2 mission it will allow for servicing of Sun observing satellites.

The trajectory analysis of Sun-Earth L1 and L2 Lagrange points was run in a manner similar to Earth-Moon L1 point and results were combined to generate curves that provide the $\Delta \mathrm{V}$ required for either an outbound or Inbound Trip. This was used to calculate fuel and consumables usage.

\section{Missions to Near-Earth Objects (NEOs)}

The idea of human exploration of near-Earth objects is not new, but began soon after human spaceflight beyond low-earth orbit became conceivable. The first concepts of piloted missions to these primitive bodies were first discussed in the 1960s and involved upgraded Saturn V rockets with an expanded version of the Apollo spacecraft to explore what were then called 'Earth-approaching asteroids.' Since then, several other studies have examined the requirements for sending humans to such primitive bodies in close proximity to Earth. NASA undertook the two most recent serious assessments, one by Langley Research Center (LaRC) completed in December 2005 initiated after the Exploration Systems Architecture Study, and one sponsored by the Constellation Program (CxP) Advanced Projects Office and undertook by a team from Ames Research Center (ARC), Johnson Space Center (JSC), and the Jet Propulsion Laboratory (JPL) completed in February 2007 (Ref. 6). The primary objective of both feasibility studies was to provide an initial assessment to determine if piloted Constellation vehicles and systems could be used to perform near-Earth object (NEO) rendezvous missions.

NEO missions could be humanity's first steps beyond the Earth-Moon system to another planetary body in the solar system. These missions would greatly increase humanity's knowledge and experience in relation to: the origin of the solar system; diversion of potentially hazardous objects (PHOs); development of safe and reliable deep space operations; development of more efficient exploration architectures; early ISRU emplacement and evaluation; and establishment of research and/or resource outposts in deep space.

To date, nearly 50 accessible NEOs have been identified (Ref. 7) for possible human exploration with round-trip mission lengths over several months. However, before conducting piloted missions, robotic precursors missions are highly desirable. A preliminary study of robotic precursor mission opportunities has been conducted for launches in years 2013 or 2014. Of the 19 large (greater than 25 meters in diameter) NEOs considered accessible for piloted missions, 12 had at least one opportunity satisfying the criteria of an arrival speed less than $1.14 \mathrm{~km} / \mathrm{s}$ and a transit time of 3 years or less. Most opportunities required Earth departure launch energies (C3) less than $16 \mathrm{~km}^{2} / \mathrm{s}^{2}$.

As the small-body database (SBDB) maintained by the Jet Propulsion Laboratory (JPL) is continually updated with new discoveries and refined orbits, it will be periodically assessed for piloted NEO mission opportunities. The most accessible NEOs in the context of piloted missions will have heliocentric orbits similar to Earth's (semi-major axis near $1 \mathrm{AU}$, low eccentricity, and low ecliptic inclination) to minimize propulsive impulses required to depart Earth, arrive at the targeted NEO, and depart the NEO for Earth return. To obtain round-trip durations less than a year with reasonably small velocity changes, piloted mission opportunities typically occur during accessible NEO encounters with Earth at minimum distances less than $0.1 \mathrm{AU}$ (15 million $\mathrm{km}$ or 37 lunar distances).

SBDB accessibility assessments initiated in June 2009 assumed the launch capability of an Ares V at Earth departure and have identified 40 to 50 potential destinations, depending on the sorting criteria used. On average, about one new accessible NEO is added to the SBDB every month. Roughly half of accessible NEOs are estimated to exceed 25 meters in diameter, and several are likely to exceed 100 meters. An illustrative human mission opportunity entails less than 200 days for its round trip (including a 10-day loiter period at the NEO destination), requires impulses totaling about $3 \mathrm{~km} / \mathrm{s}$ to arrive at and depart from the NEO destination, and achieves Earth return atmospheric entry at a geocentric inertial speed less than $11.5 \mathrm{~km} / \mathrm{s}$.

The two most recent NASA studies focused on the feasibility of mounting piloted missions to NEOs utilizing the Constellation Program hardware that was to be developed for human return to the Moon. Given the new direction 
apparent in the recent White House budget request for development of a heavy lift vehicle, piloted missions to NEOs may actually be facilitated by liberation from lunar mission architecture and performance constraints. A 156-day trajectory plot to NEA $1999 \mathrm{AO}_{10}$ and back to Earth is shown in Figure 6 as an example of a piloted mission profile that could be flown to accessible NEOs.

In general, the total mission $\Delta \mathrm{V}\left(\Delta \mathrm{V}_{\text {tot }}\right.$ is the sum of Earth departure, NEO arrival, and NEO departure $\Delta$ Vs required to perform the mission) can be reduced by a longer duration mission (i.e., 210 days), shorter stay times at the NEO (i.e., 3 to 5 days), and a possible lunar gravity assist if the NEO is in an optimal location for the piloted mission trajectory.

The crewed vehicle would require several basic capabilities in order to complete the scientific and technical objectives of the mission. The crew and spacecraft systems would need to be operationally autonomous from real-time ground control support due to distance-based communications delays. This would require significant on-board capabilities beyond what has been planned for LEO or even lunar missions. The crew would need to have the equivalent flight directors on-board to support real-time operations, EVA, and robotic systems. In addition, the crew would be a scientific vanguard to the NEOs, needing all the equipment and tools for any first explorers. This would involve equipment and techniques supporting remote sensing, deployment/re-deployment of surface experiment packages, and surface sampling. Previous ground-based observations and dedicated robotic precursor mission data should have adequately characterized the target NEO's surface and local space environment to reduce risk to the spacecraft and its assets (i.e., the crew and equipment). Hence, the majority of spacecraft operations should be able to take place in close proximity ( $\sim$ a few to several hundred meters) to the NEO. Such operations have been found to be challenging for remotely controlled spacecraft due to round trip light delay times of several tens of seconds or minutes, but should be much more tractable for human missions. Based on previous Apollo and Space Shuttle experience, the crew should be able to match the rotation of the NEO, or hover over its surface, while maintaining a stable attitude from which they can conduct a detailed scientific exploration of the surface.

Another advantage of human exploration in this realm is the capability to precisely place and re-deploy relatively small scientific and robotic packages on the NEO's surface. Packages such as remotely operated or autonomous climbers/rovers/hoppers with one or two instruments could greatly increase the amount of data obtained, helping to refine site selection for subsequent sample collection, and enhancing the diversity of samples to be collected from the surface. In situ experiments designed to test such technologies as surface anchors/tethers, drills/excavation equipment, or resource extraction equipment could also be deployed.

Undoubtedly, the biggest scientific asset that the piloted mission will have to offer is its astronaut crew, which can adapt to specific situations and adjust experiments and operations with much more flexibility than a robotic spacecraft. The crew has the added advantage of EVA and sample collection capabilities during close proximity operations. The crew's ability to rendezvous with and traverse the NEO's surface, and collect macroscopic samples in geological context from several terrains would bring a wealth of scientific information on such physical characteristics as particle size, potential space weathering effects, impact history, material properties, and near-surface densities.

\section{Mission To Asteroid (1999 AO10) In 2025/6 \\ Dotted Lines Are Projections Onto Ecliptic Plane Time Tick Labels Are 0 Hrs UTC yyyy-mm-dd}
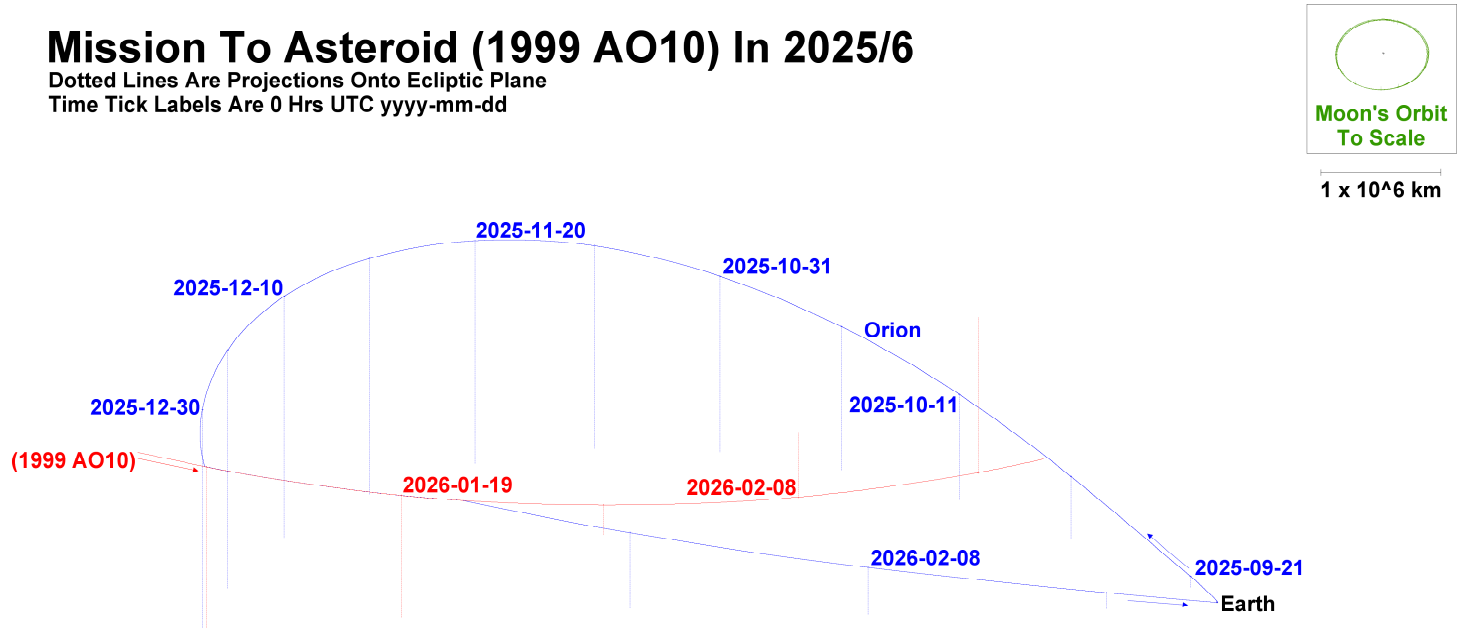

2025-09-19 @ 13:44 UTC: Launch into Earth parking orbit, $\mathrm{H}=166.7 \mathrm{~km}, \mathrm{i}=38.0^{\circ}$

2025-09-19 @ 16:07:15 UTC: Parking orbit departure, $\Delta \mathrm{v}=3.291 \mathrm{~km} / \mathrm{s}$

2026-01-08 @ 21:18 UTC: (1999 AO10) arrival, $\Delta \mathrm{v}=2.193 \mathrm{~km} / \mathrm{s}$

2026-01-08 @ 21:18 UTC: (1999 AO10) arrival, $\Delta V=2.193 \mathrm{~km} / \mathrm{s}$

2026-02-22 @ 00:02 UTC: mid-Pacific Earth return, i=14.2 ${ }^{\circ}$, speed $=11.294 \mathrm{~km} / \mathrm{s}$

Figure 6 - Earth-Centered Trajectory Plot for a Typical Piloted Near-Term NEO Mission 


\section{E. Mars Fly-by Mission and Robotic Teleoperations}

The goal of a Mars fly-by mission would be to provide a reduced-risk crewed Mars mission that provides critical knowledge and experience in preparation for an eventual Mars landing (Ref. 8). Mission durations could range from approximately $365-437$ days assuming reasonable total mission $\Delta$ Vs $(7.5 \mathrm{~km} / \mathrm{s}$ for a 437 -day mission and $9.1 \mathrm{~km} / \mathrm{s}$ for a 365-day mission) for chemical or nuclear thermal propulsive stages. A trajectory departing Earth in April 2018, resulting in a mission duration of 437 days, is shown in Figure 7. These mission durations are significantly shorter than the 900+ days required for a long-stay Mars surface mission, or a mission to explore the Martian moons, since the crew is not required to wait for the proper orbital alignment for return to Earth. Many of the technologies and operational considerations discussed previously (radiation shielding and countermeasures, regenerative life support systems, deepspace operational autonomy, etc.) would be applicable to a Mars fly-by mission. Operational verification of the spacecraft and the associated subsystems would provide critical knowledge about the long-term viability of operating them in interplanetary space. Understanding the long-term behavior and failure rates of the spacecraft subsystems could be critical before embarking on a three-year journey to Mars. The mission would also demonstrate the checkout, maintenance, and repair capability of crewed spacecraft and crew training experience during very long duration interplanetary missions.

Finally, a Mars fly-by mission could provide meaningful robotic scientific exploration of Mars or its moons. As the mission spacecraft approached Mars, previously deployed autonomous assets on Mars or its moons could transition from Earth-based and autonomous operations to a crew commanding mode via "real time" telerobotics operations.



Figure 7 - A Mars Fly-by Mission Trajectory for 2018

\section{F. Mars/Phobos Mission and Mars Sample Return}

The mission to Phobos (Ref. 9) is designed to enable the first human presence around Mars and provide access to Martian materials on Phobos. This mission would provide similar goals as the Mars fly-by mission, with the additional operational aspects of capturing into an orbit around Mars. The purpose of scientific access to Phobos is to search for 
evidence of organic compounds, and volatiles to gain an understanding of the interaction between developing or evolving biological systems and the physical environment in which this was taking place. These studies can be done by determining; the cratering history, the detailed analysis of materials, the level of organic chemistry that exists, and the relation of these factors to those on Mars, to meteorites, to interplanetary dust particles.

Phobos mission objectives included basic exploration, resource surveys to determine the existence of water, and the establishment of a science station. For this Flexible Path mission, a notional robotic Mars sample return mission was identified as a precursor that could make use of the human presence in orbit to perform final telerobotic selection of the samples. The sample return system would rendezvous with the human Mars / Phobos mission and then curated in orbit and returned with the other Phobos samples to Earth.

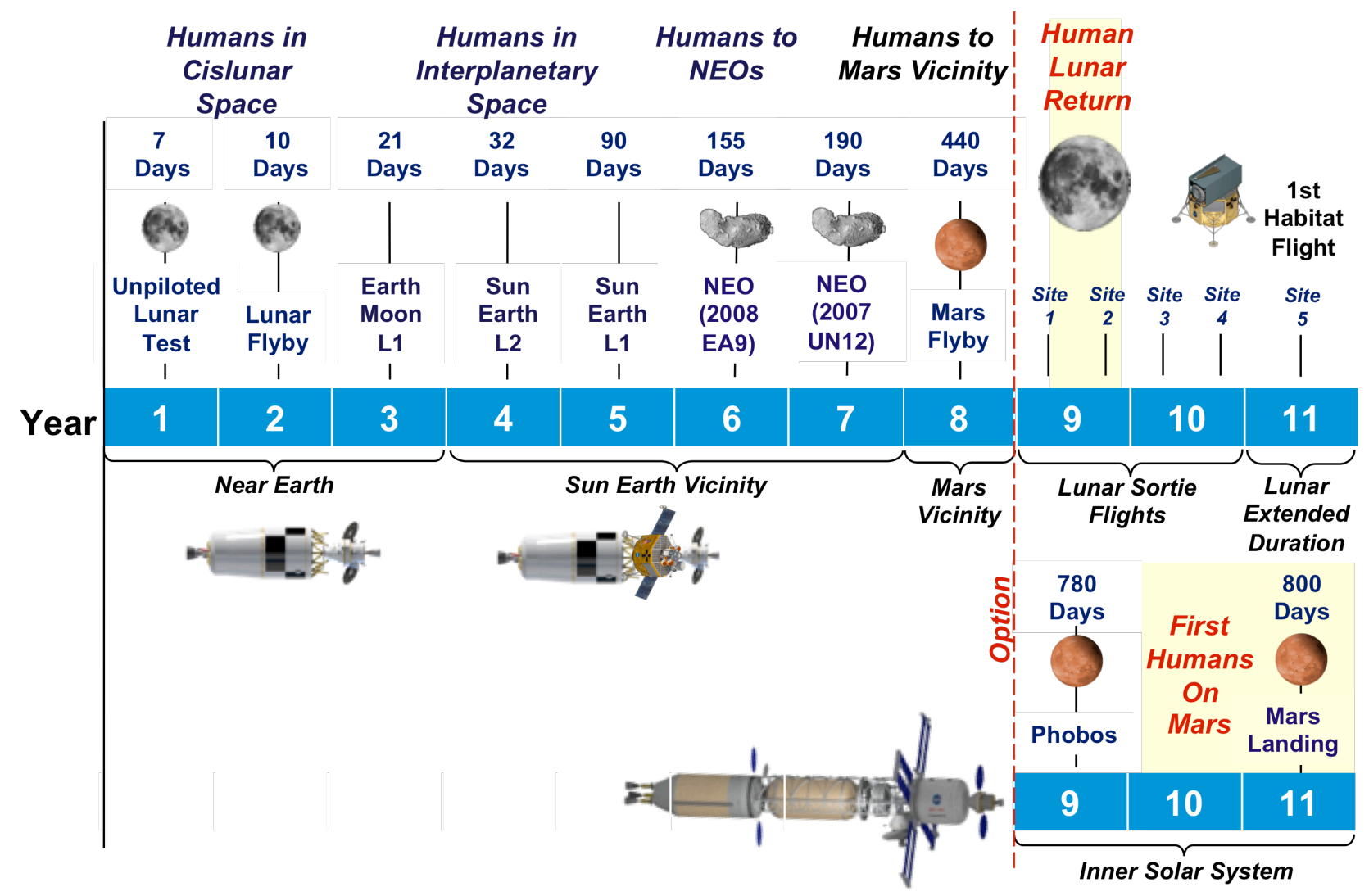

Figure 8 - Notional Flexible Path Architecture with “Off-Ramps” to Lunar or Mars Surface Exploration

\section{Mission Design and Analysis Approach}

The nature of Flexible Path philosophy requires analysis of a number of different architectures, missions, and components. None of the mission destinations identified in the Flexible Path are new. They have all been studied at one time or another in the past. The Flexible Path analysis team reviewed available datasets and leveraged as much information as possible within the scope of the Flexible Path ground rules and assumptions. The mission design and analysis process for this study included six phases that resulted in the overall performance of each mission for the given classes of in-space propulsion stage. These phases included destination identification, trajectory development, habitation and logistics sizing, payload sizing, payload packaging, and propulsive stage utilization and fueling calculations.

Destination identification and notional phasing were the first step in the Flexible Path mission development activity. Possible destinations and activities that could be performed, as well as experience or knowledge that could be gained at each location was assessed and missions were notionally ordered in incremental difficulty from both a hardware and operational standpoint. Once the destinations were identified, trajectories for each were developed or acquired with time of flight limited such that each mission to a new destination was longer than the preceding. Figure 8 illustrates the classes of missions that were considered and the final set that were down-selected to show a notional Flexible Path 
campaign manifest. It also show possible extensions to the nominal Flexible Path missions; to the Lunar or Martian surface.

The $\Delta \mathrm{V}$ required for transit to and from the destinations as well as time of flight for each mission were determined based on available trajectories. The resulting mission durations were used to size the habitation element and the logistics loads for 2-6 crew members.

\section{A. Habitation and Logistics Analysis}

Habitable and pressurized volumes required for each mission were calculated using the equation $(6.67 \mathrm{x} \ln ($ duration$)$ - 7.79) for pressurized volume per crew member for a given duration from the NASA Human-Systems Integration Handbook (Ref. 10). The required volumes for each mission were used to determine the range of volumes accommodated by the conceptual design of habitation elements to support the crew. On very early missions only the Orion CEV and a habitation module and an airlock (similar to the airlock designed for the Constellation Altair lander ascent stage) are required. As the missions begin to exceed 20 days or 4 crew members, a small transit habitat is required with a volume similar to the horizontal and vertical lunar surface habitat concepts under development by the Constellation Lunar Surface Systems Project Office. Increasing mission durations, especially with six crew, demand ever larger habitation volume up to nearly 200 cubic meters according to the handbook equation.

NASA's work on the International Space Station Trans Hab and the Mars Trans Hab was leveraged for conceptual habitation element mass and capabilities estimations that provide habitation for the crew on long-duration missions. Element sets were developed (see Figure 9) that meet the prescribed volume while keeping the number of habitation elements low. For some missions with short return times the habitation element could be segmented into two pressure vessels providing the ability to jettison one prior to trans-Earth injection (TEI) to save mass and reduce propellant requirements.

In addition to habitable volume, crew members require logistics such as food, water, clothing, medical supplies, and other consumables. The required logistics were estimated at a daily usage rate of $2.45 \mathrm{~kg} / \mathrm{day} / \mathrm{crew}$ member assuming closed-loop ECLSS. This value is based on Mars DRA version 5.0 (Ref. 11) estimates and is less than current Constellation Lunar Surface Systems Campaign Team consumables modeling for missions by approximately 15 percent for very long missions and up to 50 percent less for very short ( 10 day) missions. This difference is mitigated by an additional logistics, spares and equipment allocation of an additional $2.45 \mathrm{~kg}$ /day/crew member to provide rough order magnitude estimation prior to actual hardware design.

Equipment for use at each location was determined including an unpressurized excursion vehicle for NEO exploration and a pressurized excursion vehicle for Mars moons exploration (not shown here as it is not in the final notional mission manifest). These payloads were packaged onto a transportation system with Orion CM for re-entry, the total habitation and consumables mass versus the number of crew and duration is shown in Figure 10.

With the payload for missions of varied duration and crew size calculated, a crew size of 4 was selected for the lunar fly-by mission and a crew size of 3 was selected for all other missions. This does not preclude the inclusion of a greater number of crew, but a single value was desired for each mission to reduce the amount of data that would be presented. In addition the number of crew was held constant to show the trends in performance between the vehicle classes for each mission and over the mission set for each class of vehicle.

\begin{tabular}{|c|c|c|c|c|c|c|}
\hline \multirow{3}{*}{ Duration } & \multirow{2}{*}{$\begin{array}{c}\text { Volume per Crew } \\
\text { Member }\end{array}$} & \multicolumn{5}{|c|}{ Est. Habitable Volume for Crew sizes (m3) } \\
\hline & & 2 & 3 & 4 & 5 & 6 \\
\hline & $(6.67 \times \ln ($ duration $)-7.79)$ & & & & & \\
\hline 7 & 5.2 & 10 & 16 & 21 & 26 & 31 \\
\hline 14 & 9.8 & 20 & 29 & 39 & 49 & 59 \\
\hline 30 & 14.9 & 30 & 45 & 60 & 74 & 89 \\
\hline 100 & 22.9 & 46 & 69 & 92 & 115 & 138 \\
\hline 75 & 21.0 & 42 & 63 & 84 & 105 & 126 \\
\hline 365 & 31.6 & 63 & 95 & 126 & 158 & 189 \\
\hline 430 & 32.7 & 65 & 98 & 131 & 163 & 196 \\
\hline 430 & 32.7 & 65 & 98 & 131 & 163 & 196 \\
\hline 400 & 32.2 & 64 & 97 & 129 & 161 & 193 \\
\hline
\end{tabular}

\begin{tabular}{|c|c|c|}
\hline Elements & \multicolumn{2}{|c|}{ Total Habitable Volume } \\
\hline$=C M+A L+A S$ & $\sim 20$ & Constellation Elements \\
\hline$=\mathrm{CM}+\mathrm{AL}+\mathrm{sTH}$ & $\sim 60$ & Small Trans Hab \\
\hline$=\mathrm{CM}+\mathrm{AL}+\mathrm{mTH}$ & $\sim 100$ & Medium Trans Hab \\
\hline$=\mathrm{CM}+\mathrm{AL}+4 \mathrm{CrTH}$ & $\sim 140$ & 4 Crew Trans Hab \\
\hline$=\mathrm{CM}+\mathrm{AL}+6 \mathrm{CrTH}$ & $\sim 200$ & 6 Crew Trans Hab \\
\hline
\end{tabular}

Figure 9 - Habitation Volume and Element Configurations 


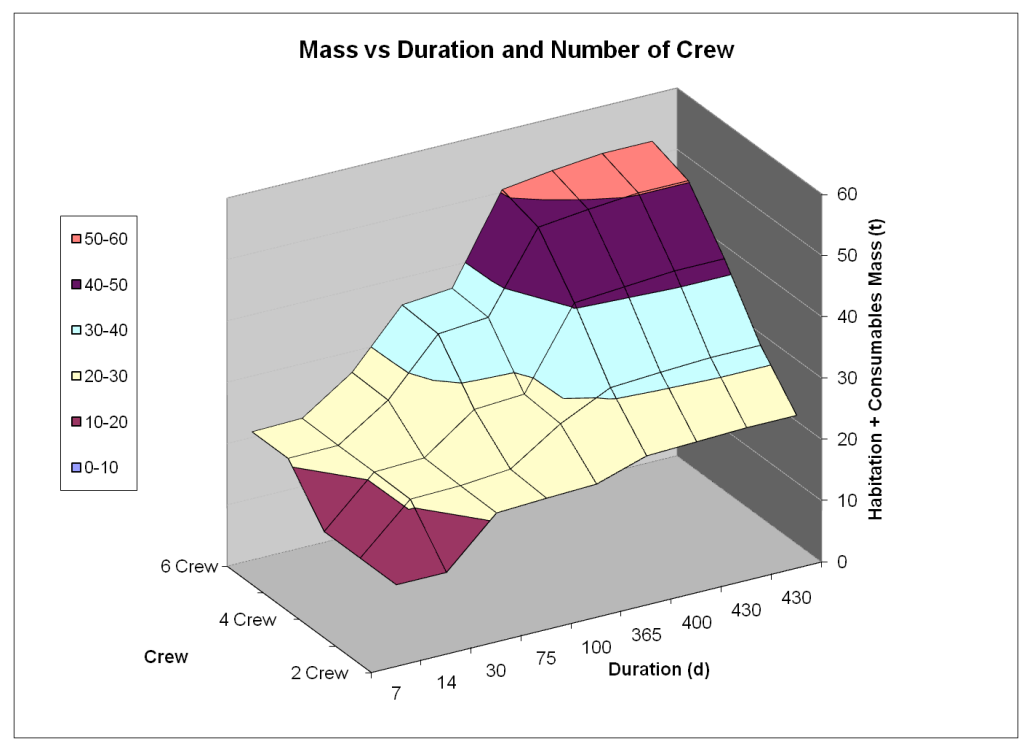

Figure 10 - Habitation \& Consumables Mass vs. Mission Duration and Number of Crew

\section{B. In Space Stage Impact Analysis}

The EA Team was directed by the Exploration Beyond LEO subcommittee to assess the impact of three classes of propulsive stages (50 t, 75 t, and 125 t) on the Flexible Path missions. The Advanced Concepts Office at Marshall Space Fight Center (MSFC) performed refuelable in-space (or Earth Departure Stage (EDS)) sizing at the request of the Exploration Beyond LEO subcommittee. These stages were designed to be flown by the differing classes of launch vehicle that were under consideration by the HSF Committee. Figure 11 shows the inert mass and maximum propellant capacity of each stage. The $25 \mathrm{t}$ EDS would be launched with $8.2 \mathrm{t}$ of propellant or cargo, the $75 \mathrm{t}$ EDS would be launched with $57.7 \mathrm{t}$ of propellant or cargo, and the $125 \mathrm{t}$ EDS would be launched with $96.5 \mathrm{t}$ of propellant or cargo. The number of stages required for each mission and propellant loading was determined based on payload for each mission. Using other Constellation components such as the Orion CM and Service Module (SM), the Flexible Path missions were designed to meet the $\Delta \mathrm{V}$ requirements for trajectories to Lagrange points, the Moon, NEOs, Mars, and Venus. Most missions were attainable using multiple launches of the larger two launch vehicles and the Ares I to deliver crew and Orion to a LEO rendezvous orbit.

The performance of the fleet of in-space propulsive stages was assessed using the rocket equation. Figure 12 shows the results for each class of in-space stage and calls out the propellant delivered, propellant required from on-orbit refueling and the payload. Columns without data represent missions that require more than two in-space stages fully fueled of that class. Interesting trends can be observed in this dataset, in particular the significant amount of un-used tankage for these missions represented by the white/transparent ranges.

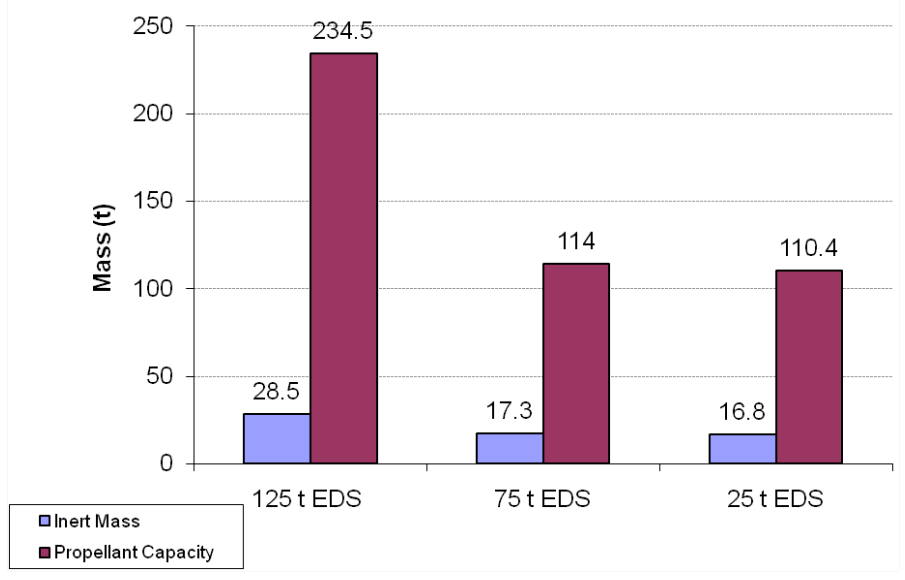

Figure 11 - In-Space Stage Inert Masses and Propellant Capacities 

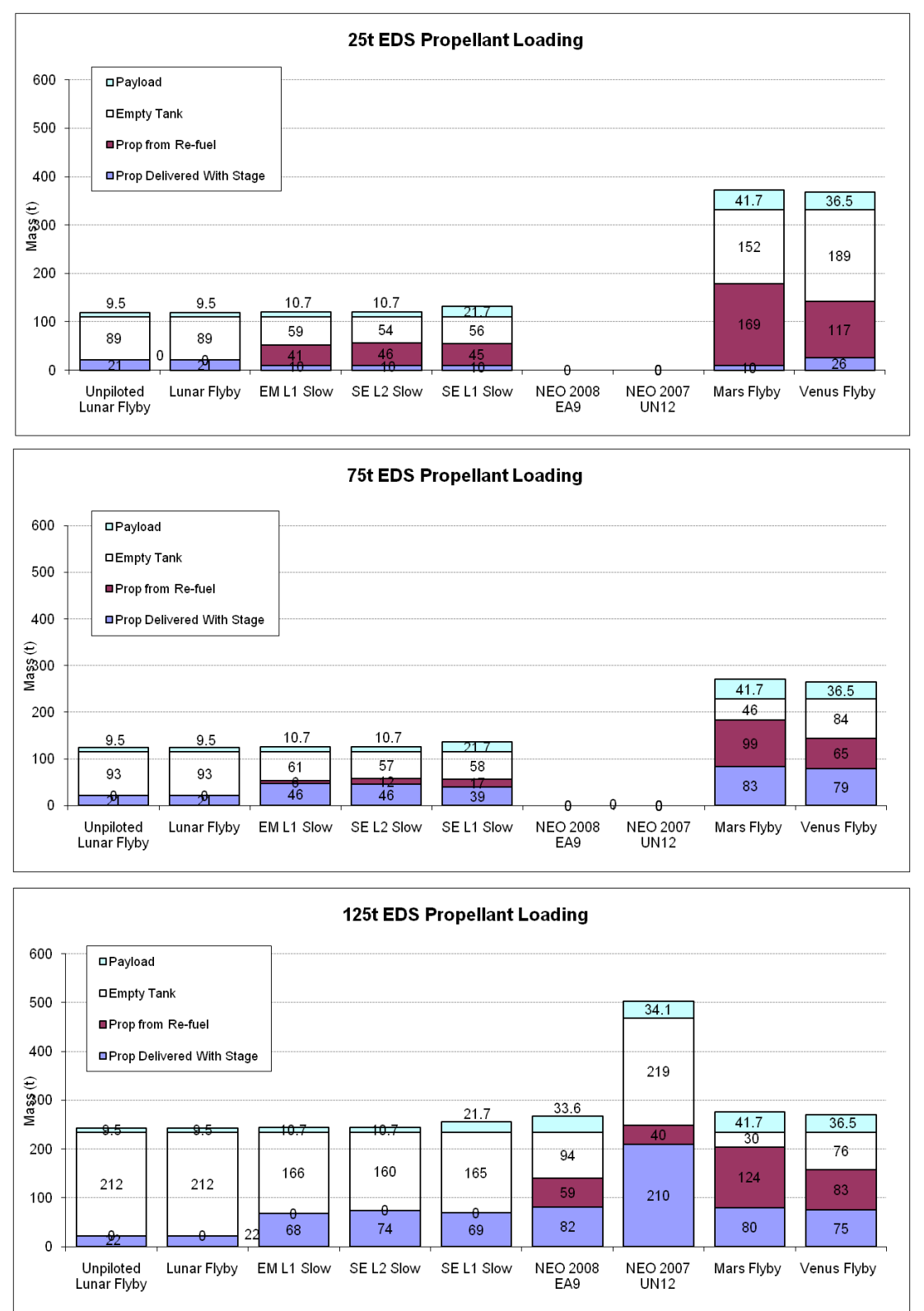

Figure 12 - EDS Propellant Loading

\section{Conclusion}

The space between Earth and Mars, while vast, is far from empty. Recent analyses supporting the Review of U.S. Human Space Flight (HSF) Plans Committee indicate that a viable exploration architecture was represented by a "Flexible Path" of ever increasing human exploration steps into interplanetary space. These steps included the exploration, for exciting scientific and important engineering reasons, of the Earth-Moon and Sun-Earth Lagrange points, our nearest interplanetary neighbors, the near-Earth objects, and initial missions to lunar and Martian orbits. Crewed visits to Flexible Path destinations can demonstrate the interplanetary capability of the next-generation piloted spacecraft, thus opening up the frontiers of the solar system in ways never before imagined for human spaceflight. There is much to be gained from understanding the inner solar system and all its destinations as NASA looks to missions to the Mars surface as its long-term goal for exploration.

The Flexible Path scenario allows for additional technology development for each mission set and allows NASA to apply new technologies as they occur to enable more and more difficult missions. This incremental approach allows for 
precursor missions and for technology demonstration to inform decision making for subsequent missions and their associated systems. The Flexible Path philosophy isn't solely a set of missions that was developed for the Beyond LEO subcommittee. Rather, it is a fundamentally different way of approaching human space exploration with phased decision points and multiple on/off ramps to various destinations that are made as critical technologies are matured and included in NASA's portfolio. An important goal of this approach is to allow for broad science objective in exploration, efficient engineering, and continual public engagement.

\section{Acknowledgments}

The authors wish to acknowledge the personnel at ARC, Glenn Research Center (GRC), LaRC, JSC, JPL, and MSFC for their assistance in finding background material and performing the analyses in support of the Flexible Path Scenario. In particular we deeply appreciate Dan Adamo's volunteer efforts in generating trajectories for the various NEO missions. Richard Mattingly (JPL) was a great assistance on discussion about the Mars sample return coupled with the Phobos mission, and John Casani was a valued "sounding board" as the Flexible Path scenario evolved.

\section{References}

${ }^{1}$ Review of U.S. Human Spaceflight Plans Committee, "SEEKING A HUMAN SPACEFLIGHT PROGRAM WORTHY OF A GREAT NATION,” U.S. Government Report, October 2009.

${ }^{2}$ NASA Technology Readiness Definitions - http://esto.nasa.gov/files/TRL_definitions.pdf

${ }^{3}$ Schwaninger, A. J. "Trajectories in the Earth-Moon Space with Symmetrical Free Return Properties.” Technical Note D-1833. Huntsville, Alabama: NASA / Marshall Space Flight Center, 1963

${ }^{4}$ Troutman, P. A., Mazanek, D. D., Stillwagon, F. H., Antol, J., Sarver-Verhey, T. R., Chato, D. J., Saucillo, R., Blue, D., Siegfried, W. H., and Carey, D., "Orbital Aggregation and Space Infrastructure Systems (OASIS)," $53{ }^{\text {rd }}$ International Astronautical Congress - World Space Congress, Houston, TX, October 10-19, 2002.

${ }^{5}$ Bret G. Drake Editor, Exploration Blueprint Data Book, NASA Johnson Space Center Report, JSC-63724, February 2007.

${ }^{6}$ Abell, P. A., Korsmeyer, D. J., Landis, R. R., Jones, T. D., Adamo, D. R., Morrison, D. D., Lemke, L. G., Gonzales, A. A., Gershman, R., Sweetser, T. H., Johnson, L. L., Lu, E. "Scientific exploration of near-Earth objects via the Orion Crew Exploration Vehicle," Meteoritics \& Planetary Science, vol. 44, Issue 12, p.1825-1836 (2009), http://meteoritics.org.

${ }^{7}$ Adamo, Daniel R., et al., "A Survey of Asteroid Destinations Accessible for Human Exploration," Journal of Spacecraft and Rockets (in peer review, 2010).

${ }^{8}$ Mazanek, D. D., Borowki, S. K., et al. "Human Mars Flyby Study,” NASA Internal Study, December 13, 2005.

${ }^{9}$ Borowski, S. K., Dudzinski, L. A., McGuire, M. L. "Vehicle and Mission Design Options for the Human Exploration of Mars/Phobos Using "Bimodal" NTR and LANTR Propulsion," NASA TM--1998-208834, AIAA-98-3883, 34 ${ }^{\text {th }}$ AIAA, ASME, SAE, and ASEE Joint Propulsion Conference, Cleveland, Ohio, July 13-15, 1998

${ }^{10}$ Pickett, L., Connolly, J., et al., "Human Integration Design Handbook (HIDH): Evolution of NASA-STD-3000," NASA Johnson Space Center, 2009.

${ }^{11}$ Mars Architecture Steering Group, Bret G. Drake Editor, "Human Exploration of Mars Design Reference Architecture 5.0," NASA Report, NASA-SP-2009-566, July 2009. 